Pacific Journal of Mathematics

A CHARACTERIZATION OF PSEUDO-ANOSOV FOLIATIONS 


\title{
A CHARACTERIZATION OF PSEUDO-ANOSOV FOLIATIONS
}

\author{
Athanase Papadopoulos and Robert C. Penner
}

Let $M$ be a closed oriented smooth surface of genus $g \geq 2$, and let $\mathscr{M} \mathscr{F}$ denote the space of equivalence classes of measured foliations on $M$. The importance of measured foliations began with Thurston's work on diffeomorphisms of surfaces: he defined the space $\mathscr{M} \mathscr{F}$ and recognized the natural action of the mapping class group on $\mathscr{M} \mathscr{F}$ as an extension of the action of this group on the Teichmüller space of $M$. In these investigations, there arose the concept of a pseudo-Anosov map which fixes a pair of transverse projective measured foliation classes on $M$, and the question evolves of recognizing the foliation classes fixed by some pseudo-Anosov map. Our main result provides a solution to this problem: we give a combinatorial characterization of these projective measured foliation classes. The combinatorial formulation of this problem uses the theory of train tracks.

\section{Introduction.}

1.1. In $\S \S 2$ and 3 of this paper, we develop a method which associates a semi-infinite combinatorial "RLS word" to a class of measured foliations. The techniques underlying these RLS words first arose in [K] (see also $[\mathbf{H P}]$ ) and depend on the machinery of train tracks; we recall the necessary material in \$1.2. The RLS word does not uniquely determine the projective measured foliation class, rather it determines exactly the subset of $\mathscr{M} \mathscr{F}$ consisting of the foliations topologically equivalent to the given foliation. (For the definitions and basic properties of measured foliations, we refer the reader to [FLP].) Section 4 contains our main results, and we completely characterize the classes of measured foliations left invariant by some pseudo-Anosov map in terms of their RLS words. Roughly, a measured foliation class is invariant under a pseudo-Anosov map if and only if it admits a preperiodic RLS word. Part of the theorem is in some sense constructive, and we describe how to find a pseudo-Anosov map fixing an invariant foliation class. This allows the description of an algorithm for producing representatives of all conjugacy classes of pseudo-Anosov map in $\$ 4.4$, wherein we also discuss some open problems and likely applications. 
1.2. Train tracks. In this section, we recall the definitions and some basic facts about train tracks that we shall make use of in this paper; for the details, we refer the reader to [HP], [P] and [T].

A train track is a branched 1-submanifold of a surface $M$, i.e., a differentiable graph embedded in $M$ with local models at the vertices given by Figure 1. We require furthermore that no complementary component of the train track is an annulus without cusps on its boundary or a disk wtih zero, one, or two cusps on its boundary.

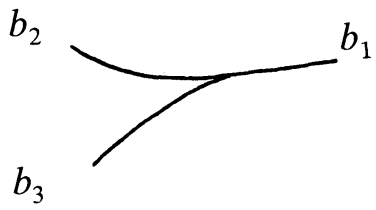

FIGURE 1

Given a train track $\tau$ with edges $a_{1}, \ldots, a_{I}$, there is a well-defined map $\varphi_{\tau}: E(\tau) \rightarrow \mathscr{M} \mathscr{F}$, where $E(\tau) \subset \mathbf{R}^{I}$ is the convex cone of nonnegative weights on the edges of $\tau$ which satisfy the condition that at each vertex as in Figure 1, the weight of $b_{1}$ is the sum of the weights of $b_{2}$ and $b_{3} . \varphi_{\tau}$ is a homeomorphism onto its image, and we denote this image by $V_{\tau}$.

We shall make use of the following terminology for the edges of a train track: an edge is large (and otherwise small) at an endpoint if each smooth arc in the train track through the endpoint intersects the interior of the edge. In Figure $1, b_{2}$ and $b_{3}$ are small, whereas $b_{1}$ is large at the given endpoint. There are thus three kinds of edges: large at both endpoints, small at both endpoints, and large at one and small at the other endpoint. We shall refer to edges of the first and second kind respectively as large and small edges.

Associated to $\tau$, there is a fibred neighborhood $N(\tau) \subset M$ equipped with a retraction $N(\tau) \searrow \tau . N(\tau)$ has singular points on its boundary, called cusps, whose local models are given by Figure 2. The set of cusps of $N(\tau)$ is in natural one-to-one correspondence with the set of vertices of $\tau$. The fibres of the retraction $N(\tau) \searrow \tau$ form a foliation $\mathscr{T}$ of $N(\tau)$ by segments called ties, and the ties that pass through the cusps are called the singular ties of $\mathscr{T} . N(\tau)$ with its foliation is well-defined up to isotopy, and $\tau$ can be regarded as a quotient space of $N(\tau)$ when every fibre is identified to a point. 


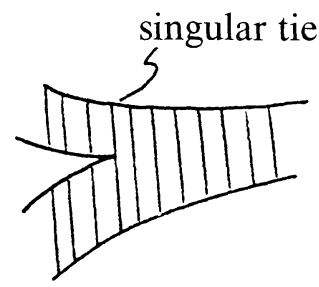

Figure 2

If $\mathscr{F}$ is an element of $\mathscr{M} \mathscr{F}$, we will usually represent $\mathscr{F}$ by a partial measured foliation of the surface $M$. This is a foliation of a subsurface of $M$ having all its singular points on the boundary of the subsurface with local models at these points given by Figure 3. If no complementary region of a partial foliation is a disk with zero or one cusp, then this partial foliation has a well-defined class in $\mathscr{M} \mathscr{F}$.

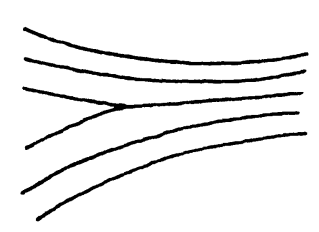

Figure 3

We say that $\mathscr{F}$ is carried by $\tau$, and we write $\mathscr{F}<\tau$, if $\mathscr{F}$ can be represented by a partial foliation which is contained in a fibred neighborhood $N(\tau)$ of $\tau$ and is transverse to the ties. The elements of $V_{\tau} \subset \mathscr{M} \mathscr{F}$ are exactly the elements of $\mathscr{M} \mathscr{F}$ that are carried by $\tau$, and a measured foliation class $\mathscr{F}$ corresponds to an element of $V_{\tau}$ which has all of its weights strictly positive if and only if $\mathscr{F}$ can be represented by a partial foliation which is transverse to the ties and whose support is equal to $N(\tau)$.

If $\sigma$ and $\tau$ are two train tracks on $M$, we say that $\sigma$ is carried by $\tau$, and we denote this relation by $\sigma<\tau$, if $\sigma$ is isotopic to a train track $\sigma^{\prime}$ which is contained in a fibred neighborhood $N(\tau)$ of $\tau$ and which is transverse to the ties. We shall make use of three elementary moves which produce from a train track $\tau$ a train track $\sigma$ which is carried by $\tau$. These moves are depicted in Figure 4(a), (b) and (c) and are called respectively a right split, a left split and a shift. If a train track $\sigma$ arises from a train track $\tau$ by splitting and shifting, then we say that $\tau$ refines to $\sigma$. 


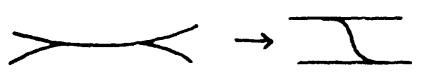

(a)

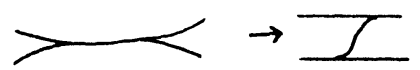

(b)

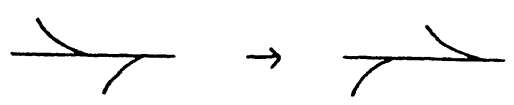

(c)

Figure 4

If $\sigma<\tau$, there is a natural linear map $\Psi: E(\sigma) \rightarrow E(\tau)$ which induces the inclusion map $V_{\sigma} \subset V_{\tau}$. The linear map $\Psi$ can be described (in a non-unique way) as the restriction of a linear map $\Psi: R^{J} \rightarrow R^{I}$, where $R^{J}$ and $R^{I}$ are respectively the vector spaces of weights (not necessarily nonnegative) on the edges of $\sigma$ and $\tau$. We describe precisely such a map $\Psi$ since we will make use of it.

Regard the fibred neighborhood $N(\tau)$ as a union of rectangles arising as the inverse images of edges of $\tau$ under the collapse $N(\tau) \searrow \tau$, where each rectangle is foliated by the ties. If $a_{1}, \ldots, a_{I}$ are the edges of $\tau$, we make a choice for each edge $a_{l}$ of a tie above an interior point of $a_{l}$; this tie is called the central tie associated to the edge $a_{i}$. Let $b_{1}, \ldots, b_{J}$ be the edges of $\sigma$, and $b_{1}^{\prime}, \ldots, b_{J}^{\prime}$ respectively the corresponding edges of $\sigma^{\prime}$, where the correspondence is given by the isotopy which carries $\sigma$ to $\sigma^{\prime}$.

Isotope $\sigma^{\prime}$ slightly so that it is in general position with respect to the central ties of $N(\tau)$ and consider the integral ( $J$ by $I$ )-matrix $A$ whose $(j, i)$ th entry is equal to the number of times the edge $b_{j}^{\prime}$ of $\sigma^{\prime}$ intersects the central tie of $N(\tau)$ which is above the edge $a_{l}$ of $\tau$. This matrix $A$ defines a linear map $\Psi: R^{J} \rightarrow R^{I}$ which induces the map $\Psi: E(\sigma) \rightarrow E(\tau)$. We again emphasize the fact that the linear map $\Psi$ from $R^{J}$ to $R^{I}$ is not canonical, but the induced linear map on the convex cones $\Psi: E(\sigma) \rightarrow$ $E(\tau)$ is independent of the choices involved in the definition of $\Psi$.

\section{Combinatorial words associated to foliations and train tracks.}

2.1. The semi-infinite word construction. Let $\mathscr{F}$ be an element of $\mathscr{M} \mathscr{F}$, $\tau$ a train track with fibred neighborhood $N(\tau)$ and $\mathscr{T}$ the foliation of $N(\tau)$ by the ties. We say that $\tau$ is suited to $\mathscr{F}$ if $\mathscr{F}$ can be represented by a partial measured foliation $F$ of support $N(\tau)$, which is transverse to the ties and has no leaves connecting cusps of $N(\tau)$. We recall that this last condition implies that each infinite half-leaf of $\mathscr{F}$ is dense in $N(\tau)$. 
Suppose that $\tau$ is suited to $\mathscr{F}$, and let $a_{1}, \ldots, a_{I}$ denote the (infinite) separatrices of $F$. The set $\left\{a_{1}, \ldots, a_{I}\right\}$ is in natural one-to-one correspondence with the set of cusps of $N(\tau)$ as well as with the set of infinite separatrices of any measured foliation representing the class $\mathscr{F}$. Let $s$ be a function from the set $\mathbf{Z}^{+}$of positive integers to the set $\{1, \ldots, I\}$. Associated to the triple $(\mathscr{F}, \tau, s)$ we wish to define:

(i) a semi-infinite word $w_{\tau}^{s}(\mathscr{F})=w_{1}, w_{2}, \ldots$ in the alphabet

$$
\left\{R_{1}, \ldots, R_{I}, L_{1}, \ldots, L_{I}, S_{1}, \ldots, S_{I}\right\} \text {. }
$$

(ii) an infinite sequence $\tau_{0}, \tau_{1}, \tau_{2}, \ldots$ of train tracks so that $\mathscr{F}$ is suited to $\tau_{n}$ for each $n=0,1,2, \ldots$, whence $\mathscr{F}$ admits a representative $F_{n}$ whose support is a fibred neighborhood $N_{n}$ of $\tau_{n}$; in this way, each cusp of $\tau_{n}$ gets a label from $\{1, \ldots, I\}$ depending on which separatrix of $F_{n}$ issues from that cusp. Furthermore, for each $n, \tau_{n+1}$ is obtained from $\tau_{n}$ by a single elementary move, which is respectively a left split, right split or shift on the cusp of $\tau_{n}$ with label $i=s(n)$ if and only if the $n$th letter $w_{n}$ of the word $w_{\tau}^{s}(\mathscr{F})$ is $L_{i}, R_{i}$ or $S_{i}$ respectively.

The sequence of train tracks $\tau_{0}>\tau_{1}>\tau_{2}>\cdots$ that the construction produces will be naturally associated with a nested sequence $N_{0} \supset N_{1} \supset$ $N_{2} \supset \cdots$ of fibred neighborhoods, so that for all $n$ the foliation of $N_{n+1}$ by the ties will be induced by the foliation of $N_{n}$ by its ties. The word $w_{\tau}^{s}(\mathscr{F})$ is called the RLS sequence (or RLS word) associated to $(\mathscr{F}, \tau, s)$.

The construction is recursive, and we begin with $\tau_{0}=\tau, N_{0}=N(\tau)$, and $F_{0}=F$. For the recursion step, we are given the train track $\tau_{n}$ with the bi-foliated neighborhood $N_{n}$ and an order on the cusps. We examine the separatrix $\alpha$ of $F_{n}$ issuing from the $s(n)$ th cusp of $N_{n}$. There are several cases indicated in Figure 5(a). These cases are distinguished by considering the first intersection of $\alpha$ with the singular ties of $N_{n}$ : case (i) (respectively (ii)) occurs when the first edge of $\tau_{n}$ that is covered by $\alpha$ (for the natural colapse $N_{n} \searrow \tau_{n}$ ) is large (recall the definition given in §1.2), and $\alpha$ travels to the left (respectively right) in $N_{n}$ after this first intersection point. Otherwise case (iii) occurs. To define the bi-foliated neighborhood $N_{n+1} \subset N_{n}$, we separate the bi-foliated neighborhood $N_{n}$ along an arc $\beta \subset \alpha$ whose initial point is at the cusp and whose endpoint lies between the first and second points of intersection of the separatrix with the singular ties. The opening operation is illustrated in Figure $5(\mathrm{~b})$, and

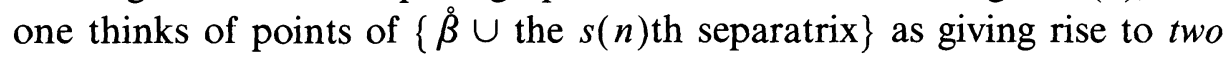
points of $\partial N_{n+1}$. Collapsing ties of $N_{n+1}$ gives the train track $\tau_{n+1} . w_{n}$ is given by $L_{s(n)}, R_{s(n)}$ or $S_{s(n)}$ if we have case (i), (ii), or (iii) respectively. The cusps of $N_{n+1}$ inherit a natural order from the order on the cusps of $N_{n}$. 


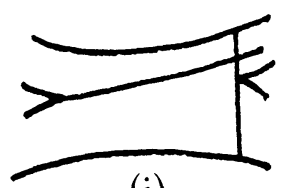

(i)

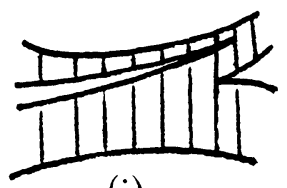

(i)

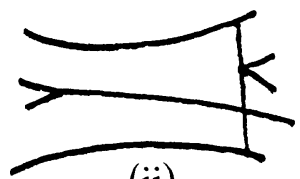

(ii)

(a)

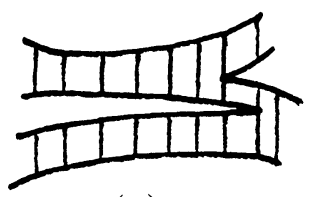

(ii)

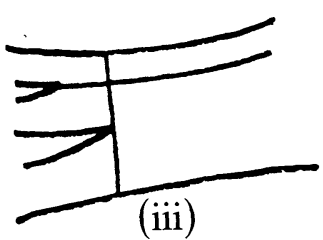

(iii)

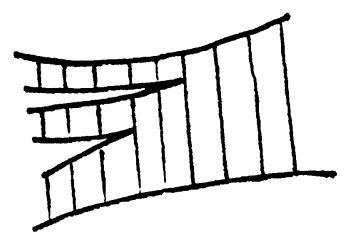

(iii)

(b)

FIGURE 5

Notice that the foliation $F_{n+1}$ induced on $N_{n+1}$ by $F_{n}$ is isotopic to $F_{n}$ even though $\tau_{n+1}$ is not isotopic to $\tau$. The leaves of the tie-foliation $\mathscr{T}_{n+1}$ on $N_{n+1}$ are subleaves of the tie foliation $\mathscr{T}_{n}$ on $N_{n}$. The train tracks $\tau_{n}$, $n \geq 1$, are said to arise from $\tau$ by refinement along the separatrices of $\mathscr{F}$.

2.2. Refinement of train tracks suited to foliations. We would like next to associate a finite combinatorial word to the following situation: $\mathscr{F}<\sigma$ $<\tau$, and $\tau$ is suited to $\mathscr{F}$. This finite word describes a finite number of elementary moves by which $\tau$ refines to $\sigma$. We require some technical results.

2.1. Lemma. Suppose $\mathscr{F}<\sigma<\tau$, where $\sigma$ is a train track contained in $N(\tau)$ transverse to the ties, and $\tau$ is suited to $\mathscr{F}$. There is then a fibred neighborhood $N(\sigma)$ of $\sigma$ with $N(\sigma) \subset \stackrel{\circ}{N}(\tau)$ and a family $\left\{\Gamma_{l}\right\}_{1}^{I}$ of arcs disjointly embedded in $N(\tau)-\stackrel{\circ}{N}(\sigma)$ transverse to the ties so that $\left\{\partial \Gamma_{i}\right\}_{1}^{I}$ gives a pairing of cusps of $N(\tau)$ and cusps of $N(\sigma)$. For a fixed $N(\sigma) \subset \stackrel{\circ}{N}(\tau)$ the family $\left\{\Gamma_{i}\right\}_{1}^{I}$ is unique up to isotopy fixing the endpoints.

Proof. Insofar as $\sigma \subset N(\tau)$ is transverse to the ties, we may choose a tie neighborhood $N(\sigma)$ with $N(\sigma) \subset \stackrel{\circ}{(\tau)}$, where the ties of $N(\sigma)$ are subarcs of the ties of $N(\tau)$. Fix a cusp of $N(\tau)$ and consider the corresponding singular tie $t$. There is a point of $t \cap \partial N(\sigma)$ on each side of the cusp, for otherwise, as $\mathscr{F}<\sigma$, there would exist a representative $F$ of $\mathscr{F}$ contained in $N(\tau)$ transverse to the ties which does not pass through every tie of $N(\tau)$. This would contradict the uniqueness of the system of weights on the edges of $\tau$ representing $\mathscr{F}$. 


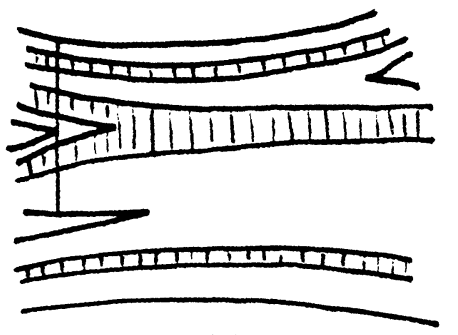

(a)

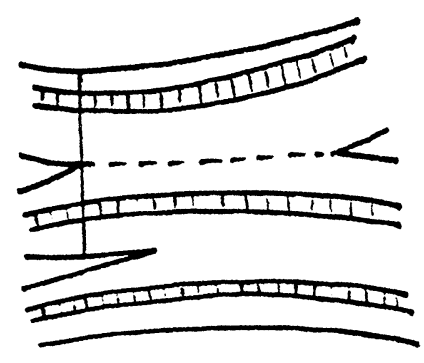

(b)

FigURE 6

Consider the edges of $\partial N(\sigma)$ which intersect $t$ nearest the cusp on either side as in Figure 6(a). We claim that these edges of $\partial N(\sigma)$ coalesce at a cusp of $N(\sigma)$ as in Figure 6(a). If not, then these edges must traverse distinct paths and so eventually diverge as in Figure 6(b). In this case, consider a representative $F$ of $\mathscr{F}$ supported in $N(\sigma)$ and transverse to the ties. From this foliation, we can construct another representative supported on $N(\tau)$ transverse to the ties which has a leaf which connects two cusps of $N(\tau)$. This contradicts the unicity of the measure on $\tau$ describing $\mathscr{F}$.

We have shown that edges of $\partial N(\sigma)$ nearest cusps of $N(\tau)$ along singular ties coalesce at cusps of $N(\sigma)$; conversely, one sees easily that each cusp of $N(\sigma)$ is associated in this way to a cusp of $N(\tau)$. There is thus a pairing of the cusps of $N(\sigma)$ and the cusps of $N(\tau)$, and each pair in this pairing determines a region $W_{i}, i=1, \ldots, I$, of $N(\tau)-\stackrel{\circ}{N}(\sigma)$ bounded by subarcs of edges of $\partial N(\sigma)$ and singular ties of $N(\tau)$ (see Figure 7). Collapsing subties of these regions gives the required collection of arcs.

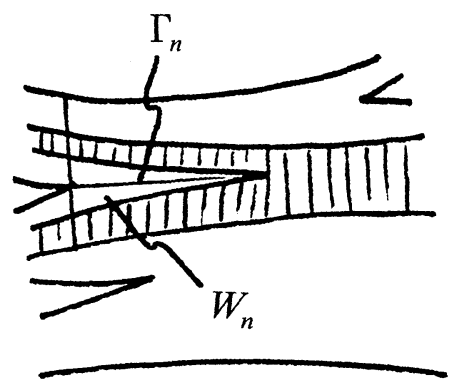




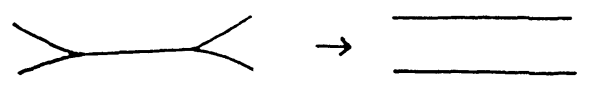

FIGURE 8

If $\sigma$ and $\tau$ are two train tracks, recall that $\tau$ refines to $\sigma$ if $\sigma$ can be obtained from $\tau$ by a finite sequence of splits and shifts. There is another move, called a collision, which we might perform on a train track and which is illustrated in Figure 8. If a train track $\sigma$ arises from a train track $\tau$ by splitting, shifting and colliding, then we say $\tau$ refines to $\sigma$ with collisions.

2.2. Proposition. Suppose $\mathscr{F}<\sigma<\tau$ with $\sigma \subset N(\tau)$ transverse to the ties and with $\tau$ suited to $\mathscr{F}$. In this situation, $\tau$ refines to $\sigma$ (without collisions $), \sigma$ is suited to $\mathscr{F}$, and $\sigma$ may be isotoped along the ties of $N(\tau)$ to a train track $\sigma^{\prime}$ so that $\sigma^{\prime}$ arises from $\tau$ by refinement along the separatrices of $\mathscr{F}$. In fact, the refinement to $\sigma^{\prime}$ may be done by refining first along separatrix one, then separatrix two,.., and finally along separatrix $I$.

Proof. Let $W_{l}, i=1, \ldots, I$, be the regions of $N(\tau)-\stackrel{\circ}{N}(\sigma)$ constructed in Lemma 2.1, and consider the quotient $N^{\prime}$ of $N(\sigma)$ obtained by collapsing the subties on each region $W_{l}$. Collapsing ties of $N^{\prime}$ gives a train track $\tau^{\prime}<\tau$ which refines (perhaps with collisions) to $\sigma$ and hence carries $\mathscr{F}$. Furthermore, the switches of $\tau^{\prime}$ coincide exactly with the cusps of $N(\tau)$ by construction.

We claim that the collapse of ties of $N(\tau)$ restricts to a homeomorphism of $\tau^{\prime}$ to $\tau$, and it suffices to show that exactly one branch of $\tau^{\prime}$ intersects each tie of $N(\tau)$. If not, then we can find a pair of adjacent intersections. The corresponding adjacent edges of $N(\tau)-\tau^{\prime}$ eventually diverge by construction, so we can find an arc in $N(\tau)-\tau^{\prime}$ transverse to the ties connecting cusps of $N(\tau)$, which is impossible as before. Thus, $\tau^{\prime}$ is homeomorphic to $\tau$, and so $\tau$ itself refines to $\sigma$. Since the switches of $\tau$ and $\sigma$ are in one-to-one correspondence by Lemma 2.1, this refinement involves no collisions.

Now, consider the compatible tie neighborhood $N(\sigma) \subset \stackrel{ }{N}(\tau)$ of $\sigma$ with its foliation representing $\mathscr{F}$. The collapse of subties induces a foliation on $N^{\prime}$ which also represents $\mathscr{F}$. Expanding $N^{\prime}$ along ties to agree with $N(\tau)$ (without changing the topology of $N^{\prime}$ ) gives a new foliaton of support $N(\tau)$ representing $\mathscr{F}$, so this new foliation differs from a given representative of $\mathscr{F}$ with support $N(\tau)$ by isotopy along the ties. It follows that $\sigma$ may be isotoped along the ties of $N(\tau)$ to some $\sigma^{\prime}$ 
so that $\sigma^{\prime}$ arises from $\tau$ by refinement along the separatrices of $\mathscr{F}$; it follows easily that $\mathscr{F}$ is suited to $\sigma$.

Finally, rather than collapsing the regions $W_{i}, i=1, \ldots, I$, all at once, we might collapse them in reverse order: first $W_{I}$, then $W_{I-1}, \ldots$, and finally $W_{1}$. We require here that the $W_{i}$ are pairwise disjoint. The last assertion of the proposition then follows.

2.3. The zipper construction. Let $\mathscr{F}, \tau$ and $\sigma$ be as in $\S 2.2$; we now associate to this triple:

(i) a finite sequence $\tau=\tau_{0}>\tau_{1}>\cdots>\tau_{L}=\sigma$ of train tracks with each $\tau_{i}$ suited to $\mathscr{F}$, together with a sequence $N_{0}>N_{1}>\cdots>N_{L}$ of bi-foliated neighborhoods with an ordering on the cusps

(ii) a finite RLS-word $w_{\tau}(\sigma)=w_{1} \cdots w_{L}$, where the $i$ th letter $w_{i}$ describes the carrying $\tau_{l-1}>\tau_{i}$.

Let $\beta_{l}$ the subarc of the $i$ th separatrix of the foliation on $N(\tau)$ representing $\mathscr{F}$ along which we refine to pass from $\tau$ to the train track $\sigma^{\prime}$ guaranteed by Proposition 2.2. Set $i=0$ and $\tau_{0}=\tau$. Recursively, let $p_{i+1}$ be the total number of intersections of $\beta_{i+1}$ with singular ties of $N\left(\tau_{l}\right)$, and let $\tau_{i+1}$ be the refinement of $\tau_{i}$ along the subarc $\beta_{i+1}$ of the $(i+1)$ th separatrix.

We generate in this way a sequence $p_{1}, \ldots, p_{I}$ of nonnegative integers and define

$$
\begin{gathered}
s:\left\{0,1, \ldots, \sum_{1}^{I} p_{i}\right\} \rightarrow\{1, \ldots, I\} \\
: j \mapsto k, \text { if } \sum_{1}^{k-1} p_{i}<j \leq \sum_{1}^{k} p_{i} .
\end{gathered}
$$

Finally, perform the first $\sum_{1}^{I} p_{t}$ stages of the recursion in the construction of $\$ 2.1$ to generate the associated train tracks, the bi-foliated neighborhoods, and finite RLS-word $w_{\tau}(\sigma)$.

3. Convergent RLS sequences. Let $\mathscr{F}, \tau$ and $s$ be as in $\S 2.1$, $w_{\tau}^{s}(\mathscr{F})$ the associated RLS-sequence, and $\tau_{0}, \tau_{1}, \ldots$ the associated sequence of train tracks. We say that the semi-infinite word $w_{\tau}^{s}(\mathscr{F})$ is convergent if it contains infinitely many $R$ or $L$ letters.

Choose some representative $(F, \mu)$ of $\mathscr{F}(\mu$ being the transverse measure on $F$ ), and let $\Delta_{\mathscr{F}}$ denote the subset of $\mathscr{M} \mathscr{F}$ consisting of the classes of measured foliations which admit representatives of the form $\left(F, \mu^{\prime}\right)$, where $\mu^{\prime}$ is some invariant transverse measure on $F$. The set $\Delta_{\mathscr{F}}$ does not depend on the choice of representative $(F, \mu)$ of $\mathscr{F}$. This section 
is devoted to proving

3.1. THEOREM. If the word $w_{\tau}^{s}(\mathscr{F})$ is convergent, then

$$
\bigcap_{i=0}^{\infty} V_{\tau_{l}}=\Delta_{\mathscr{F}} \text {. }
$$

REMARK. If the word is not convergent, then it is clear that $\bigcap_{i=0}^{\infty} V_{\tau_{i}}$ strictly contains $\Delta_{\mathscr{F}}$, since a shift on the train track $\tau_{i}$ does not change the set $V_{\tau_{l}} \subset \mathscr{M} \mathscr{F}$.

For the proof of the theorem, we first remark that the construction of the $\tau$, does not depend on the transverse measure but only on the trajectory structure of the separatrices of $\mathscr{F}$ relative to $\tau$. It follows that $\Delta_{\mathscr{F}} \subset \cap_{i=0}^{\infty} V_{\tau_{i}}$.

To prove the reverse inclusion, we require some lemmas.

3.2. LemMA. Let $\mathscr{F}$ and $\mathscr{G}$ be two elements of $\mathscr{M} \mathscr{F}$ carried by a train track $\tau$ with corresponding systems of weights $\left(x_{k}\right)$ and $\left(y_{k}\right), k=1, \ldots, K$, respectively. Then we have the inequality

$$
i(\mathscr{F}, \mathscr{G}) \leq \sum_{k=1}^{K} x_{k} y_{k} .
$$

$(i(\cdot, \cdot)$ denotes the intersection function on $\mathscr{M} \mathscr{F} \times \mathscr{M} \mathscr{F}$; see $[$ FLP]. $)$

Proof. We may suppose that all the $x_{k}$ are strictly positive; the general case then follows by continuity of the intersection function. Represent $\mathscr{F}$ by a foliation $F$ of a fibred neighborhood $N(\tau)$ of $\tau$ so that $F$ is transverse to the ties. Recall that the vertices of $\tau$ are in natural one-to-one correspondence with the cusps of $N(\tau)$.

We embed $\tau$ in $N(\tau)$ in such a way that:

each vertex of $\tau$ is at the corresponding cusp of $N(\tau)$;

each edge of $\tau$ is either in a leaf of $F$ or transverse to $F$ and is embedded in $N(\tau)$ in one of the five ways indicated in Figure 9. Consider now the system of weights $\left(y_{k}\right)$ on the train track $\tau$. Edge number $k$ of $\tau$ has transverse measure (with respect to $F$ ) at most $x_{k}$. The inequality

$$
i(\mathscr{F}, \mathscr{G}) \leq \sum_{k=1}^{K} x_{k} y_{k}
$$

then follows easily from the definition of the intersection function, first by supposing that $\mathscr{G}$ is the class of a simple closed curve, and then by continuity. 

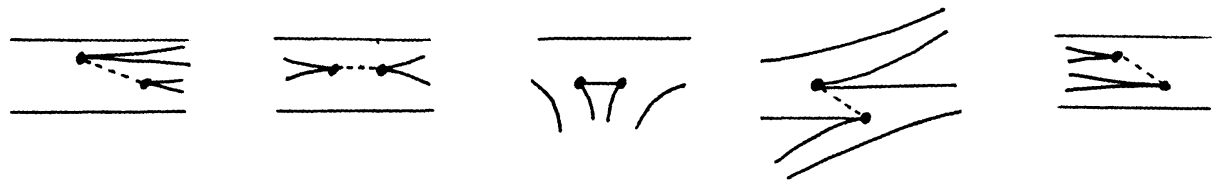

Figure 9

3.3. Proposition. With the same hypotheses as in Theorem 3.1, if $\mathscr{G}$ is an element of $\bigcap_{n=0}^{\infty} V_{\tau_{n}}$, then $i(\mathscr{F}, \mathscr{G})=0$.

Proof. For each $n=0,1,2, \ldots$, let $\left(x_{k}^{n}\right)$ and $\left(y_{k}^{n}\right), k=1, \ldots, K$, be the systems of weights on the train track $\tau_{n}$ for $\mathscr{F}$ and $\mathscr{G}$ respectively. We want to show that as $n$ tends to infinity, $\sum_{k=1}^{K} x_{k}^{n} y_{k}^{n}$ tends to zero. Since each train track $\tau_{n+1}$ is obtained from its predecessor $\tau_{n}$ by splitting or shifting, we have

$$
\max _{k}\left\{x_{k}^{n}\right\} \leq \max _{k}\left\{x_{k}^{0}\right\}
$$

and

$$
\max _{k}\left\{y_{k}^{n}\right\} \leq \max _{k}\left\{y_{k}^{0}\right\}
$$

for all $n=1,2, \ldots$. To prove the proposition, it is therefore sufficient to prove:

3.4. Lemma. As $n$ tends to infinity, we have $\max _{k}\left\{x_{k}^{n}\right\}$ tends to zero.

Proof. Recall that in the construction of the sequence $\tau_{0}, \tau_{1}, \ldots$ given in $\$ 2.1$, at each stage there is a foliation $F_{n}$ in the class $\mathscr{F}$ which is supported in a fibred neighborhood $N_{n}$ of the train track $\tau_{n} . F_{n}$ is obtained from $F_{n-1}$ by opening the fibred neighborhood $N_{n-1}$ along a separatrix segment; there is also a foliation $\mathscr{T}_{n}$ by the ties which is transverse to $F_{n}$. We want to show that the largest transverse $F_{n}$-measure of the ties of $\mathscr{T}_{n}$ tends to zero as $n$ tends to infinity.

Put a Lebesgue-equivalent transverse measure $\nu$ of full support on the foliation $\mathscr{T}$ so that the total transverse $\nu$-measure of a branch of $\tau$ is equal to one. In this way, the total transverse $\nu$-measure between two successive singular ties in a positive integer. For each $n$, there is an induced transverse measure on $\mathscr{T}_{n}$.

If we can show that there exists a sequence of successive separatrix segments of $F$ which are involved in the refining process so that the total transverse $\nu$-measure of this sequence is infinite, then the lemma will be 
proved. Indeed, each semi-infinite separatrix of $F$ is dense in the support of $F$ as well as in the support of each foliation $F_{n}$. If we now take a segment of arbitrarily small $F_{n}$-measure $\varepsilon$ at the center of each tie of each central tie of $N_{n}$, then these segments will be separated at some later stage $n^{\prime}$ in the refining process; it follows that

$$
\max _{k}\left\{x_{k}^{n \prime}\right\} \leq \frac{1}{2} \max _{k}\left\{x_{k}^{n}\right\}+\varepsilon .
$$

Thus, to prove Proposition 3.3 it remains to prove the

3.5. LEMMA. There exists a chain of separatrix segments of $F$ whose total transverse $\nu$-measure is infinite which are involved (in the sense above) in the construction of the word $w_{\tau}^{s}(\mathscr{F})$.

Proof. Recall the operation of opening the neighborhood described in §2.1. For the purpose of the proof, choose a small number $\varepsilon>0$ and a sequence of positive numbers $\varepsilon_{i}, i=1,2, \ldots$, satisfying $\varepsilon=\sum_{l=0}^{\infty} \varepsilon_{i}$. We specify that at each stage $i$ of the opening process, the endpoint of the separatrix segment $\beta$ involved is at some $\nu$-distance at most $\varepsilon_{i}$ from the first intersection point with a singular tie (see Figure 10). With this convention, each separatrix segment involved in the refinement has $\nu$ transverse measure either greater than $1-\varepsilon$ or less than $\varepsilon$. One sees from the construction that whenever the segment has transverse $\nu$-measure less than $\varepsilon$, the corresponding move on the train track is necessarily a shift. By the convergence of the word $w_{\tau}^{s}(\mathscr{F})$, there are infinitely many splits involved and also therefore infinitely many separatrix segments of $\nu$-measure greater than $1-\varepsilon$. This proves Lemma 3.5 and hence Proposition 3.3.

To finally prove Theorem 3.1, it remains to show

3.6. LemmA. If $\tau$ is suited to $\mathscr{F}$ and if $\mathscr{G}$ satisfies $i(\mathscr{F}, \mathscr{G})=0$ with $\mathscr{G} \in V_{\tau}$, then $\mathscr{G} \in \Delta_{\mathscr{F}}$.

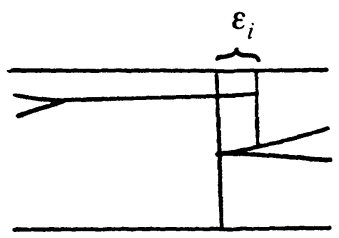


Proof. Suppose that $\mathscr{G} \in \Delta_{\mathscr{F}}$. Since $i(\mathscr{F}, \mathscr{G})=0$, each representative of $\mathscr{G}$ has a subfoliation which is disjoint from a representative of $\mathscr{F}$. As $\mathscr{F}$ and $\mathscr{G}$ are carried by $\tau$, there exists a measured foliation $H$ in the fibred neighborhood $N(\tau)$ of $\tau$ which is transverse to the ties and which is the union of two subfoliations $F$ and $F^{\prime}$ which are disjoint except on the frontier of their support and where $F$ is in the equivalence class $\mathscr{F}$.

Since $\tau$ is suited to $\mathscr{F}$, the separatrices of $F$ are in one-to-one correspondence with the cusps of $N(\tau)$. Thus, $F^{\prime}$ has no separatrices and is therefore a foliation by closed curves. On the other hand, the fact that $\tau$ is suited to $\mathscr{F}$ implies that the complement in $N(\tau)$ of $F$ is a union of annuli, each having one boundary component in $\partial N(\tau)$ and the other one in the frontier of the support of $F$.

Now, $F$ cannot have closed leaves, so the foliation induced on such a complementary annulus by the ties is a standard foliation as in Figure 11. Therefore there cannot be a smooth closed curve embedded transverse to the ties in such an annulus. Thus follows Lemma 3.6.

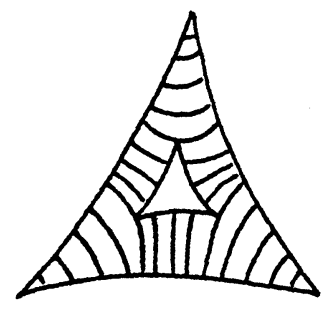

FiguRE 11

The proof of Theorem 3.1 is now complete.

\section{RLS sequences and pseudo-Anosov homeomorphisms.}

4.1. Train tracks and pseudo-Anosov homeomorphisms. Recall that a homeomorphism $\Psi$ of $M$ is called pseudo-Anosov if there exist a pair of transverse measured foliations $\left(F^{u}, \mu^{u}\right)$ and $\left(F^{s}, \mu^{s}\right)$, called respectively the unstable and stable foliations of $\Psi$, and a real number $\lambda>1$ called the dilatation of $\Psi$, so that

$$
\Psi\left(F^{u}, \mu^{u}\right)=\left(F^{u}, \lambda \mu^{u}\right)
$$

and

$$
\Psi\left(F^{s}, \mu^{s}\right)=\left(F^{s}, \lambda^{-1} \mu^{s}\right) .
$$

This section is intended to make explicit some well-known relations between pseudo-Anosov homeomorphisms and train tracks. We prove the 
following

4.1. THEOREM. If $\Psi$ is a pseudo-Anosov homeomorphism and $\mathscr{F}^{u} \in \mathscr{M} \mathscr{F}$ is the class of its unstable foliation, then there exists a train track $\tau$ suited to $\mathscr{F}^{u}$ so that $\Psi \tau<\tau$. Furthermore, if $N(\tau)$ is a fibred neighborhood of $\tau$, then $\Psi \tau$ is isotopic to a train track $\tau^{\prime}$ in $N(\tau)$ which is transverse to the ties so that the matrix describing the linear map from the space of weights on the edges of $\tau^{\prime}$ to the space of weights on the edges of $\tau$ is primitive irreducible (i.e., has some iterate all of whose entries are strictly positive).

Proof. Let $F^{u}$ and $F^{s}$ denote the unstable and stable foliations of $\Psi$ respectively. For each singular point $P$ of $F^{u}$, consider a small disk $D_{P}$ around $P$ having as many cusps on its boundary as the number of separatrices of $F^{u}$ at $P . D_{P}$ is to be embedded in the surface as in Figure 12 , where the cusps of $\partial D_{P}$ are on the $F^{u}$-separatrices and the boundary of $D_{P}$ is transverse to $F^{s}$. Consider the subsurface $M^{\prime}=M-\cup_{P} \stackrel{\circ}{D}_{P}$ of $M$ with the foliation $\mathscr{T}$ induced by $F^{s}$. By the density of the leaves of $F^{s}$, each leaf of $\mathscr{T}$ is homeomorphic to a segment and has its endpoints on the boundary of $M^{\prime}$. By general position, we can assume that there are no leaves of $\mathscr{T}$ connecting two singular points on $\partial M^{\prime}$. We can regard $M^{\prime}$ as the fibred neighborhood $N(\tau)$ of a train track $\tau$ with $\mathscr{T}$ the foliation by the ties; the singular ties correspond to the leaves of $\mathscr{T}$ which pass through the singular points of $\partial M^{\prime}$. By "blowing up" $F^{u}$ at each singular point and pushing $F^{u}$ in $N(\tau)$ along $F^{s}$-leaves (see Figure 13), we obtain a partial foliation $F$ in the class $\mathscr{F}^{u}$ of $F_{u}$ whose support is $N(\tau)$ and which is transverse to $\mathscr{T}$. $F^{u}$ has no saddle connections, and therefore $F$ has no leaves connecting singular points of $N(\tau)$. We conclude that $\tau$ is

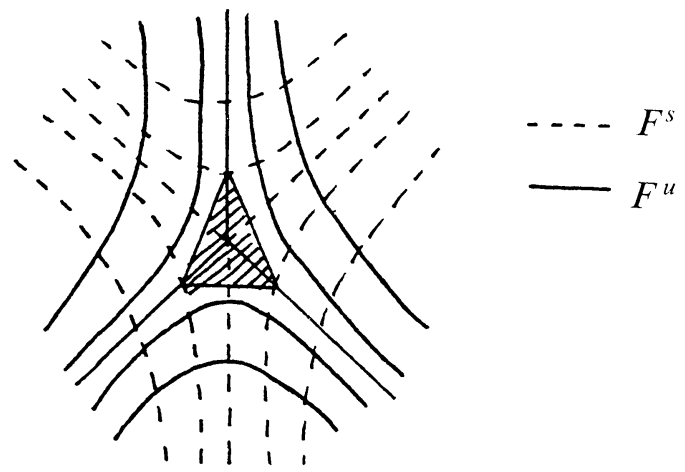

Figure 12 

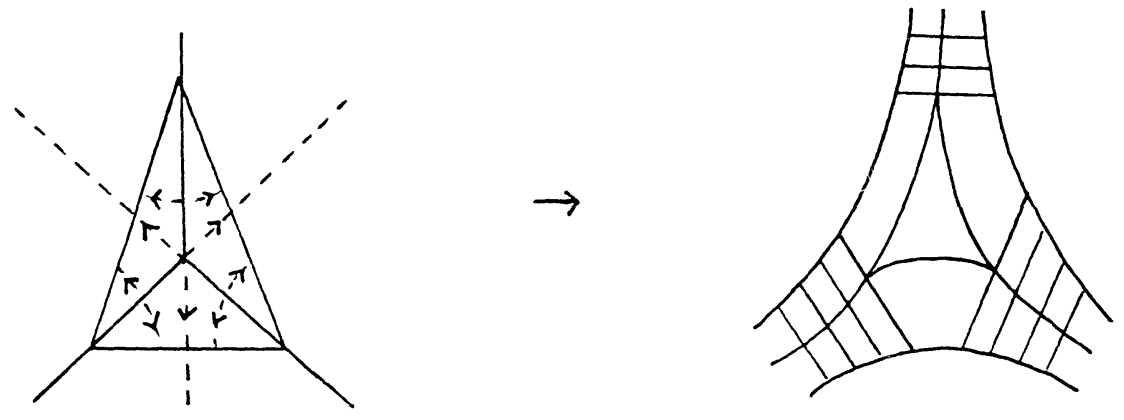

FIGURE 13

suited to $\mathscr{F}^{u}$. We represent $\tau$ as a train track embedded in the interior of $N(\tau)$ transverse to the ties so that each edge of $\tau$ contains a segment which is in a leaf of $F^{u}$.

Consider now the image train track $\Psi(\tau)$. Insofar as $\Psi$ preserves the foliation $F^{s}, \Psi(\tau)$ is transverse to this foliation and does not pass through any singular point of $F^{s}$. In each disk $D_{P}$, we can therefore push $\Psi(\tau)$ along the $F^{s}$-leaves to get a train track $\tau^{\prime}$, which is isotopic to $\Psi(\tau)$ and contained in $N(\tau)$ transverse to the ties. Thus, $\Psi(\tau)<\tau$.

On the other hand, by density of the leaves of $F^{u}$ in $M$, the fact that each edge of $\tau$ contains a segment in a leaf of $F^{u}$, and by the expansion property of $F^{u}$-leaves under $\Psi$, there exists a positive integer $l$ so that if $\tau_{l}$ is the train track obtained from $\Psi^{\prime}(\tau)$ by pushing it inside $N(\tau)$ as before, then each edge of $\tau_{l}$ intersects each tie of $\mathscr{T}$. This implies that the matrix describing the linear map from the vector space of weights on the branches of $\tau_{l}$ to the vector space of weights on the branches of $\tau$ has all of its entries strictly positive. We conclude that $\Psi(\tau)$ satisfies the requirements of the theorem.

4.2. Foliations invariant by pseudo-Anosov homeomorphisms. Recall that a foliation $F$ is said to be arational if there is no closed curve in the surface $M$ which is the union of segments joining singular points of $F$. (This is equivalent to the definition given in [FLP], Expose 9, §III.) If this condition is satisfied by $F$, then it is also satisfied by every other foliation which is Whitehead equivalent to $F$. We can therefore use this terminology for classees in $\mathscr{M} \mathscr{F}$.

4.2. TheOREM. Let $\mathscr{F}$ be an arational element of $\mathscr{M} \mathscr{F}$ and $\tau$ a train track suited to $\mathscr{F}$ which admits a preperiodic and convergent $R L S$-sequence $w_{\tau}^{s}(\mathscr{F})$ ( for some choice of function $s$ ). There then exists a pseudo-Anosov homeomorphism $\Psi$ of $M$ so that $\mathscr{F}$ is the class of the unstable foliation of $\Psi$. 
Proof. The assumption that the word $w_{\tau}^{s}(\mathscr{F})$ is preperiodic means that it admits an expression of the form $w_{\tau}^{s}(\mathscr{F})=u v v v \cdots$, where $u$ is a string of $k$ letters and $v$ a string of $p$ letters from the alphabet. Let $\left(\tau_{l}\right)_{l \geq 0}$ be the sequence of train tracks associated with $w_{\tau}^{s}(\mathscr{F})$. Consider the subsequence $\left(\tau_{k+l p}\right)_{l \geq 0}$ of train tracks, for $p=1,2, \ldots$ Up to conjugacy, there exist only finitely many train tracks on $M$. Therefore, there are positive integers $I$ and $J$ and a diffeomorphism $\Psi$ of $M$ so that $\Psi\left(\tau_{k+I p}\right)=\tau_{k+(I+J) p}$. It follows that $\Psi\left(\tau_{k+I p}\right)<\tau_{k+I p}$. The corresponding linear map

$$
A: E\left(\tau_{k+I p}\right) \rightarrow E\left(\tau_{k+I p}\right)
$$

induces a map on the set of rays in $E\left(\tau_{k+I p}\right)$ which has a fixed point by the Brouwer theorem. There thus exists an element $\mathscr{F}^{\prime} \in V_{\tau k+I p}$ so that $\Psi \mathscr{F}^{\prime}=\lambda^{\prime} \mathscr{F}^{\prime}$, where $\lambda^{\prime}$ is some positive real number. The measured foliation class $\mathscr{F}^{\prime}$ is represented by a system of nonnegative weights on the branches of $\tau_{k+I p}$, and this system of weights is identified with an eigenvector of the matrix $A$ with eigenvalue $\lambda^{\prime}$.

We wish to prove that $\mathscr{F}^{\prime} \in \bigcap_{i=0}^{\infty} V_{\tau_{i}}$. The sequence of train tracks $\left(\tau_{h+I+I J p}\right)_{l \geq 0}$ are all topologically conjugate, and as the sequence of elementary moves which allow one to pass from a train track in this sequence to its successor is independent of $l$ by preperiodicity, the matrices which describe the sequence of carryings $\tau_{k+I+(l+1) J_{p}}<\tau_{k+I+l J p}$ are identical; these matrices thus have the common eigenvector which describes the measured foliation class $\mathscr{F}^{\prime}$. Therefore $\mathscr{F}^{\prime} \in \bigcap_{l=0}^{\infty} V_{\tau_{k}+I+l J_{p}}$, whence $\mathscr{F}^{\prime} \in \bigcap_{l=0}^{\infty} V_{\tau_{i}}$.

By Theorem 3.1, $\mathscr{F}^{\prime}$ is topologically equivalent to $\mathscr{F}$, and therefore $\mathscr{F}^{\prime}$ is also arational. By [FLP], Exposé $9, \S \mathrm{IV}$ and V, $\Psi$ is isotopic to either a pseudo-Anosov map or to a periodic map. The latter case cannot occur since the action of $\Psi$ on $V_{\tau_{k}+I p}$ sends this cone strictly into itself by the convergence of the word $w_{\tau}^{s}(\mathscr{F})$. Thus, $\Psi$ is isotopic to a pseudoAnosov map, and the theorem is proved.

Since the unstable foliation of a pseudo-Anosov map is uniquely ergodic ([FLP], Exposé 12), we have the following

4.3. COROLlary. If an arational measured foliation class admits a preperiodic and convergent $R L S$ word, then it is uniquely ergodic.

REMARK. It is an interesting and unsolved problem to exactly characterize the uniquely ergodic measured foliation classes in terms of RLS sequences. 
We have the following converse to Theorem 4.2.

4.4. THEOREM. Let $\mathscr{F} \in \mathscr{M} \mathscr{F}$ be the class of the unstable foliation of a pseudo-Anosov map $\Psi$ of $M$. There exists a train track $\tau$ suited to $\mathscr{F}$ and a function s from $\mathbf{Z}^{+}$to the ordered set of infinite separatrices of $\mathscr{F}$ so that the word $w_{\tau}^{s}(\mathscr{F})$ is convergent and periodic.

Proof. By Theorem 4.1, there exists a train track $\tau$ suited to $\mathscr{F}$ so that $\Psi(\tau)<\tau$. Let $N(\tau)$ be a fibred neighborhood of $\tau$ and $\tau^{\prime}$ a train track isotopic to $\Psi(\tau)$ contained in $N(\tau)$ transverse to the ties. By Lemma 2.1, there is a finite set of $\operatorname{arcs}\left\{\Gamma_{i}\right\}_{1}^{I}$, transverse to the ties which match the vertices of $\tau$ and $\tau^{\prime}$. By replacing $\Psi$ by one of its iterates, we can suppose that this pairing of vertices is the same as the pairing given by the map followed by isotopy.

Consider now the finite word $w_{\tau}\left(\tau^{\prime}\right)$ obtained from the zipper construction (of \$2.3) applied to the present situation; we have the corresponding finite sequence $\tau_{0}=\tau_{1}, \tau_{1}, \ldots, \tau_{L}=\tau^{\prime}$ of train tracks. Construct the semi-infinite word by repeating $w_{\tau}\left(\tau^{\prime}\right)$, and consider the corresponding infinite sequence $\tau_{0}, \tau_{1}, \ldots, \tau_{L}, \tau_{L+1}, \ldots$ of train tracks. For each positive integer $k$, the train track $\tau_{(k+1) L}$ is topologically conjugate to $\tau_{k L}$ by the pseudo-Anosov map $\Psi$. By the dynamics of pseudo-Anosov maps on $\mathscr{M} \mathscr{F}$, the intersection of the nested sequence $V_{\tau_{i}}$ consists exactly of the element $\mathscr{F} \in \mathscr{M} \mathscr{F}$.

Finally, by construction each element in this intersection admits the semi-infinite word $w_{\tau}\left(\tau^{\prime}\right) w_{\tau}\left(\tau^{\prime}\right) \cdots$, which is periodic. That this word is convergent follows from the fact that the nested intersection of the $V_{\tau_{i}}$ consists of the singleton $\{\mathscr{F}\}$.

4.5. A characterization of pseudo-Anosov foliations carried by a given track. We can in fact prove the following generalization of Theorem 4.4.

4.5. THEOREM. Let $\mathscr{F} \in \mathscr{M} \mathscr{F}$ be the equivalence class of the unstable foliation of a pseudo-Anosov map of $M$. Then for every train track $\sigma$ suited to $\mathscr{F}$, there exists a function $s$ from $\mathbf{Z}^{+}$to the ordered set of infinite separatrices of $\mathscr{F}$ so that the word $w_{\sigma}^{s}(\mathscr{F})$ is convergent and preperiodic.

Proof. Fix a neighborhood $N(\sigma)$ of $\sigma$ with a foliation $\mathscr{T}^{\prime}$ by the ties, and let $F^{\prime}$ be a representative of the class $\mathscr{F}$ whose support is equal to $N(\sigma)$ and which is transverse to the ties. Consider the train track $\tau$ given by Theorem 4.3 with $N(\tau)$ its fibred neighborhood, $F$ the foliation of 
$N(\tau)$ representing $\mathscr{F}$ and $\tau_{0}, \tau_{1}, \ldots$ the sequence of train tracks corresponding to the periodic word $w_{\tau}^{s}(\mathscr{F})$. As before, the fact that the two representatives $F$ and $F^{\prime}$ have no leaves connecting singular points implies that these two partial foliations are isotopic. Let $\Phi$ be a diffeomorphism of $M$ isotopic to the identity so that $\Phi F=F^{\prime}$.

From the construction of the infinite sequence of train tracks associated with a RLS word, it is easy to see that as $i$ goes to infinity, the train track $\tau_{l}$ can be embedded in $N(\tau)$ in such a way that the angles made by the edges of $\tau_{i}$ with the leaves of $F$ tend uniformly to zero. This is essentially a consequence of the fact that the refinement operation "follows the leaves" of $F$.

Now, by compactness of $M, \Phi$ has bounded distortion. Therefore, for $i$ sufficiently large, $\Phi\left(\tau_{l}\right)$ makes small angles with $F^{\prime}$ and is transverse to $\mathscr{T}^{\prime}$. It follows that $\Phi \tau_{t}<\sigma$. Take such a large $i$ and consider finally a finite combinatorial RLS word $w$ given by the zipper construction ( $\$ 2.4)$, which describes the elementary moves required to pass from $\sigma$ to $\tau_{i}$. This finite word $w$ followed by the semi-infinite word induced by $w_{\tau}^{s}(\mathscr{F})$ on $\tau_{i}$ then defines the preperiodic semi-infinite word $w_{\sigma}^{s}(\mathscr{F})$.

4.6. Concluding remarks. In Theorems 4.2 and 4.4 we have given a complete characterization of which classes of measured foliations on $M$ are left invariant by some pseudo-Anosov map of $M$. Theorem 4.5 further characterizes the invariant foliation classes which are carried by a given train track. Furthermore, the argument of Theorem 4.4 gives a method for producing a pseudo-Anosov map fixing a given invariant foliation class. Thus, our endeavors give a procedure for constructing all conjugacy classes of pseudo-Anosov maps: enumerate representatives $\left\{\tau_{i}\right\}^{K}$, of all the conjugacy classes of all the train tracks filling $M$ (i.e., train tracks all of whose complementary regions are disks); compute the symmetry groups $\left\{G_{l}\right\}^{K}$, of these tracks; enumerate all the periodic RLS sequences on each $\tau_{i}$ so that the associated linear maps on measures are primitive irreducible, and employ the method of Theorem 4.3 to generate a sequence of pseudo-Anosov maps $\left\{\Psi_{i}\right\}_{i \geq 1}$; finally, consider $\{g \circ \Psi: \Psi$ arises from a RLS sequence on $\tau_{i}$ and $\left.g \in G_{i}\right\}$.

We further remark that there is an estimate which says roughly that the dilatation of a pseudo-Anosov map is large if the period of the RLS word is large. This estimate is derived in our paper [PP] and allows one to bound the Teichmüller geometry of moduli space viz. the number of Teichmüller geodesics of moduli space of a fixed length. In this latter regard, it would be useful (and of independent interest) to characterize the 
periods which occur for pseudo-Anosov maps (i.e., the periods which determine primitive irreducible linear maps on measures).

\section{REFERENCES}

[FLP] A. Fathi, F. Laudenbach and V. Poenaru, Travaux de Thurston sur les surfaces, Astérisque, XXX (1979), 66-67.

[HP] J. Harer and R. C. Penner, Combinatorics of train tracks, Annals of Math Studies, to appear (1986).

[K] S. Kerckhoff, Simplicial systems for interval exchange maps and measured foliations, Ergodic Theory and Dynamical Systems, 5 (1985), 257-271.

[P] A. Papadopoulos, Réseaux Ferroviaires, Diffeomorphismes Pseudo-Anosov et Automorphismes Symplectiques de l'Homologie, Publ. Math. d'Orsay, 83-03.

[PP] A. Papadopoulos and R. C. Penner, Enumerating pseudo-Anosov foliations, preprint (1986).

[T] W. Thurston, The Geometry and Topology of Three-Manifolds, Princeton University Lecture Notes (1978).

Received June 16, 1986 and in revised form January 28, 1987.

Université Louis Pasteur

7 RUE RENE DESCARTES

67084 Strasbourg, France

AND

UNIVERSITY OF SOUTHERN CALIFORNIA

LOS ANGELES, CA 90089

U.S.A. 



\section{EDITORS}

\author{
V. S. VARADARAJAN \\ (Managing Editor) \\ University of California \\ Los Angeles, CA 90024 \\ HeRbert Clemens \\ University of Utah \\ Salt Lake City, UT 84112 \\ R. FINN \\ Stanford University \\ Stanford, CA 94305
}

\author{
HERMANN FLASCHKA \\ University of Arizona \\ Tucson, AZ 85721 \\ RAMESh A. GANGOLLI \\ University of Washington \\ Seattle, WA 98195 \\ VAUGHAN F. R. JONES \\ University of California \\ Berkeley, CA 94720
}

\author{
ROBION KIRBY \\ University of California \\ Berkeley, CA 94720 \\ C. C. MOORE \\ University of California \\ Berkeley, CA 94720 \\ HAROLD STARK \\ University of California, San Diego \\ La Jolla, CA 92093
}

\section{ASSOCIATE EDITORS}
R. ARENS
E. F. BECKENBACH
B. H. NEUMANN
F. WOLF
K. YOSHIDA
(1906-1982)

\section{SUPPORTING INSTITUTIONS}

UNIVERSITY OF ARIZONA
UNIVERSITY OF BRITISH COLUMBIA
CALIFORNIA INSTITUTE OF TECHNOLOGY
UNIVERSITY OF CALIFORNIA
MONTANA STATE UNIVERSITY
UNIVERSITY OF NEVADA, RENO
NEW MEXICO STATE UNIVERSITY
OREGON STATE UNIVERSITY

\author{
UNIVERSITY OF OREGON \\ UNIVERSITY OF SOUTHERN CALIFORNIA \\ STANFORD UNIVERSITY \\ UNIVERSITY OF HAWAII \\ UNIVERSITY OF TOKYO \\ UNIVERSITY OF UTAH \\ WASHINGTON STATE UNIVERSITY \\ UNIVERSITY OF WASHINGTON
}

The Supporting Institutions listed above contribute to the cost of publication of this Journal, but they are not owners or publishers and have no responsibility for its content or policies.

Mathematical papers intended for publication in the Pacific Journal of Mathematics should be in typed form or offset-reproduced (not dittoed), double spaced with large margins. Please do not use built up fractions in the text of the manuscript. However, you may use them in the displayed equations. Underline Greek letters in red, German in green, and script in blue. The first paragraph must be capable of being used separately as a synopsis of the entire paper. In particular it should contain no bibliographic references. Please propose a heading for the odd numbered pages of less than 35 characters. Manuscripts, in triplicate, may be sent to any one of the editors. Please classify according to the scheme of Math. Reviews, Index to Vol. 39. Supply name and address of author to whom proofs should be sent. All other communications should be addressed to the managing editor, or Elaine Barth, University of California, Los Angeles, California 90024.

There are page-charges associated with articles appearing in the Pacific Journal of Mathematics. These charges are expected to be paid by the author's University, Government Agency or Company. If the author or authors do not have access to such Institutional support these charges are waived. Single authors will receive 50 free reprints; joint authors will receive a total of 100 free reprints. Additional copies may be obtained at cost in multiples of 50 .

The Pacific Journal of Mathematics is issued monthly as of January 1966. Regular subscription rate: $\$ 190.00$ a year (5 Vols., 10 issues). Special rate: $\$ 95.00$ a year to individual members of supporting institutions.

Subscriptions, orders for numbers issued in the last three calendar years, and changes of address should be sent to Pacific Journal of Mathematics, P.O. Box 969, Carmel Valley, CA 93924, U.S.A. Old back numbers obtainable from Kraus Periodicals Co., Route 100, Millwood, NY 10546.

The Pacific Journal of Mathematics at P.O. Box 969, Carmel Valley, CA 93924 (ISSN 0030-8730) publishes 5 volumes per year. Application to mail at Second-class postage rates is pending at Carmel Valley, California, and additional mailing offices. Postmaster: send address changes to Pacific Journal of Mathematics, P.O. Box 969, Carmel Valley, CA 93924.

PUBLISHED BY PACIFIC JOURNAL OF MATHEMATICS, A NON-PROFIT CORPORATION Copyright (c) 1987 by Pacific Journal of Mathematics 


\section{Pacific Journal of Mathematics}

\section{Vol. 130, No. $2 \quad$ October, 1987}

Bernhard Banaschewski, J. L. Frith and C. R. A. Gilmour, On the congruence lattice of a frame ............................ 209

Paul S. Bourdon, Density of the polynomials in Bergman spaces ........2215

Lawrence Jay Corwin, Approximation of prime elements in division

algebras over local fields and unitary representations of the multiplicative group

Stephen R. Doty and John Brendan Sullivan, On the geometry of extensions of irreducible modules for simple algebraic groups

Karl Heinz Dovermann and Reinhard Schultz, Surgery of involutions

with middle-dimensional fixed point set ....................2 275

Ian Graham, Intrinsic measures and holomorphic retracts ............ 299

John Robert Greene, Lagrange inversion over finite fields . . . . . . . . . . . 313

Kristina Dale Hansen, Restriction to $\mathrm{GL}_{2}(\mathrm{O})$ of supercuspidal

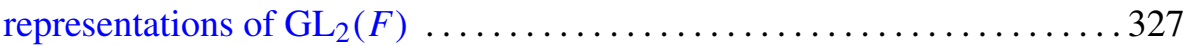

Kei Ji Izuchi, Unitary equivalence of invariant subspaces in the polydisk ....351

A. Papadopoulos and R. C. Penner, A characterization of pseudo-Anosov

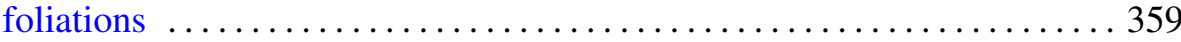

Erik A. van Doorn, The indeterminate rate problem for birth-death

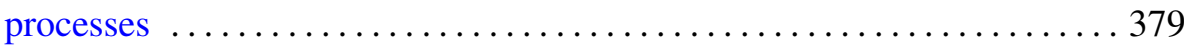

Ralph Jay De Laubenfels, Correction to: "Well-behaved derivations on

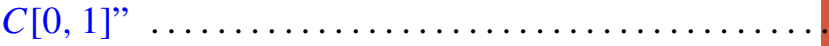

Robert P. Kaufman, Correction to: "Plane curves and removable sets" . . . . 396

Richard Scott Pierce and Charles Irvin Vinsonhaler, Correction to:

"Realizing central division algebras" 